\title{
Electric quadrupole moment of the neutron-rich ${ }^{33} \mathrm{Al}$
}

\author{
T. Nagatomo ${ }^{1, a}$, K. Shimada ${ }^{2}$, K. Asahi ${ }^{3}$, D.L. Balabanski ${ }^{4}$, J.M. Daugas ${ }^{5}$, M. Depuydt ${ }^{6}$, M. De Rydt ${ }^{6}$, \\ L. Gaudefroy ${ }^{5}$, S. Grévy ${ }^{7}$, Y. Hasama ${ }^{3}$, Y. Ichikawa ${ }^{1}$, D. Kameda ${ }^{1}$, P. Morel $^{5}$, L. Perrot ${ }^{8}$, C. Stödel ${ }^{7}$, J.C. Thomas ${ }^{7}$, \\ W. Vanderheijden ${ }^{6}$, N. Vermeulen ${ }^{6}$, P. Vingerhoets ${ }^{6}$, A. Yoshimi ${ }^{1}$, G. Neyens ${ }^{6}$, and H. Ueno ${ }^{1}$ \\ 1 RIKEN Nishina Center Wako, 351-0198 Saitama, Japan \\ 2 Tohoku University, 6-3 Aoba, Aramaki, Aoba-ku, Sendai, 980-8578 Miyagi, Japan \\ 3 Tokyo Institute of Technology, 2-12-1 Ookayama, Meguro-ku, 152-8550 Tokyo, Japan \\ ${ }^{4}$ Institute for Nuclear Research and Nuclear Energy, Bulgarian Academy of Sciences, 1784 Sofia, Bulgaria \\ ${ }^{5}$ CEA/DIF/DPTA/PN, BP 12, F-91680 Bruyeres le Chatel, France \\ ${ }^{6}$ K.U. Leuven, Instituut voor Kern- en Stralingsfysica, 3001 Leuven, Belgium \\ 7 Grand Accelerateur National d'Ions Lourds, BP 55027, F-14076 Caen codex 5, France \\ 8 Institut de Physique Nuclelaire, F-91406 Orsay, France
}

Received: 31 December 2008 / Revised: 19 August 2009

Published online: 16 October 2009 - (C) Società Italiana di Fisica / Springer-Verlag 2009

Communicated by C. Signorini

\begin{abstract}
The nuclear electric quadrupole moment ( $Q$ moment) of the neutron-rich nucleus ${ }^{33} \mathrm{Al}(I=5 / 2$, $T_{1 / 2}=41 \mathrm{~ms}$ ) has been measured by the $\beta$-ray detected nuclear quadrupole resonance $(\beta$-NQR $)$ method for the first time. The ${ }^{33} \mathrm{Al}$ nucleus is considered to be on the border of the island of inversion within which a significant intrusion of the $p f$ orbits occurs across the $N=20$ shell gap and the intrusion causes an anomalous enhancement of the $Q$ moment. Polarized ${ }^{33} \mathrm{Al}$ nuclei were produced from ${ }^{36} \mathrm{~S}(77.5 \mathrm{MeV} / u)$ beams through the fragmentation process and separated by LISE fragment separator at GANIL. The ${ }^{33} \mathrm{Al}$ nuclei were implanted into a $\mathrm{Al}_{2} \mathrm{O}_{3}$ single-crystal plate and the $\beta$-NQR spectrum was successfully obtained.
\end{abstract}

PACS. 21.10.Ky Electromagnetic moments - 76.60.Gv Quadrupole resonance - 21.60.Cs Shell model 25.70.Mn Projectile and target fragmentation

\section{Introduction}

The nuclear shell structure evolution which progresses toward the neutron drip line is an intriguing problem for both theorists and experimentalists. Nuclei which are located near the stability line have the closed shell structure at the magic numbers $(2,8,20,28$, etc.) and it has been considered to be a universal truth for all nuclei. However, surprising experimental discoveries in $\gamma$-decay studies of ${ }^{32} \mathrm{Mg}$, e.g. low location of the first $2^{+}$level $[1,2]$ and sizably enhanced $B(E 2)$ strength [3], were considered to reflect an anomalous deformation of the ground state in spite of the neutron closed shell structure of $N=20$. Theoretical analysis [4] also suggested that the shell gap between the $s d$-shell and the $f p$-shell reduced for neutronrich nuclei in a limited region $(Z=10-12, N=20-22)$, and the $2 p-2 h$ excitation played an important role to describe their nuclear structures. The limited region is known as the island of inversion. Moreover, anomalous

\footnotetext{
a e-mail: nagatomo@riken.jp
}

ground-state properties around the original island of inversion were revealed from recent studies of the electromagnetic moments of $\mathrm{Na}[5,6]$ and $\mathrm{Mg}[7,8]$ isotopes. Because the neutron-rich $\mathrm{Al}$ isotopes are considered to be on the border of the island of inversion, the nuclear structure of these $\mathrm{Al}$ isotopes is interesting to reveal the shell structure evolution. The $\mu\left({ }^{30,32} \mathrm{Al}\right)[9]$ and $Q\left({ }^{31,32} \mathrm{Al}\right)[10$, 11] were reported by RIKEN and Tokyo Tech groups and $\mu\left({ }^{31-34} \mathrm{Al}\right)[12,13]$ by Leuven group. The $\mu\left({ }^{33,34} \mathrm{Al}\right)$ seem to deviate from the conventiodenal $s d$-shell model prediction. From the Monte Carlo shell model calculation (SDPF-M), the probability of the $f p$-shell intruder configuration is predicted to be $64 \%$ in the ground state of ${ }^{33} \mathrm{Al}[14]$. The $Q$ moment is more sensitive to the intrusion phenomena than the $\mu$ moment because the $2 p$ - $2 h$ excitation causes the anomalous deformation. In the present work, the $Q$ moment of ${ }^{33} \mathrm{Al}\left(I=5 / 2, T_{1 / 2}=41 \mathrm{~ms}\right)$ has been measured by the $\beta$-ray detected nuclear quadrupole resonance $(\beta$-NQR) in a high magnetic field for the first time. In this report, we will describe the details of the measurement. 


\section{Experiments}

The experiment was performed at GANIL. ${ }^{33} \mathrm{Al}$ nuclei were produced through the projectile fragmentation process in heavy-ion collisions of ${ }^{36} \mathrm{~S}(77.5 \mathrm{MeV} / u)$ and Be nuclei. The thickness of the production target was $1212 \mu \mathrm{m}$. The produced ${ }^{33} \mathrm{Al}$ were separated by two dipole magnets and a wedge-shaped energy degrader of Be $(1068 \mu \mathrm{m}$ thick) installed in the high-resolution fragment separator LISE. The purity of ${ }^{33} \mathrm{Al}$ was typically $75 \%$. To induce nuclear spin polarization, ${ }^{33} \mathrm{Al}$ fragments with higher momenta than the peak momentum and deflection angle of $2^{\circ} \pm 1^{\circ}$ relative to the primary beam were selected. After these selections, the $1.3 \mathrm{kcps}$ polarized ${ }^{33} \mathrm{Al}$ nuclei were transported to our NMR setup installed at the end of the beam line at the primary beam current of $2.1 e \mu \mathrm{A}$. The ${ }^{33} \mathrm{Al}$ nuclei were implanted into a cubic $\mathrm{Si}$ single crystal and a non-cubic $\alpha-\mathrm{Al}_{2} \mathrm{O}_{3}$ single crystal (corundum) for the $\beta$-NMR and $\beta$-NQR measurements, respectively. The crystal was set at the center of the NMR setup. The static magnetic field $B_{0}$ of 0.5000 tesla was applied parallel to the polarization to maintain the polarization and to induce the Larmor precession. To induce NMR of ${ }^{33} \mathrm{Al}$, a strong radio frequency (rf) was applied perpendicular to the $B_{0}$. Asymmetric $\beta$-ray emission from the polarized ${ }^{33} \mathrm{Al}$ was detected by two sets of the plastic-scintillationcounter telescopes which consisted of three thin plastic scintillation counters. These detectors were placed above (up) and below (down) the crystal. The up/down ratio of the $\beta$-ray counts $R_{0}$ were proportional to the polarization $P$ as

$$
\begin{aligned}
R_{0} & =\zeta(1+A P) /(1-A P) \\
& \simeq \zeta(1+2 A P),
\end{aligned}
$$

where $\zeta$ is the geometrical asymmetry including the $\beta$-ray detection efficiencies and $A$ is the asymmetry parameter of the $\beta$-ray angular distribution. By means of the adiabatic fast passage (AFP) method of the NMR technique, the polarization was flipped when the strong rf-field with the resonance frequency was applied. In the case of the NMR of ${ }^{33} \mathrm{Al}$ in $\mathrm{Si}$, the resonance frequency was corresponding to the Larmor frequency $\nu_{L}=g \mu_{N} B_{0} / h$. Taking the ratio of the up/down ratio $R$ obtained by applying the resonance frequency to the $R_{0}$, the large asymmetry change was observed around the resonance frequency as

$$
\begin{aligned}
\text { asymmetry change } & \equiv R / R_{0}-1 \\
& \simeq-4 A P .
\end{aligned}
$$

In the case of the $\beta$-NQR measurement, the electric field gradient $q$ of the implantation site of ${ }^{33} \mathrm{Al}$ in the non-cubic $\alpha-\mathrm{Al}_{2} \mathrm{O}_{3}$ induced the quadrupole interaction with the $Q$ moment of ${ }^{33} \mathrm{Al}$, and the Larmor frequency $\nu_{L}$ split into five frequencies as shown in fig. 1. These five frequencies were corresponding to the two-level transition between neighboring magnetic sublevels $m$ and $m+1$ and were given as

$$
f_{m, m+1} \simeq \nu_{L}-\nu_{Q}\left(3 \cos ^{2} \theta-1\right)(2 m+1) / 4,
$$

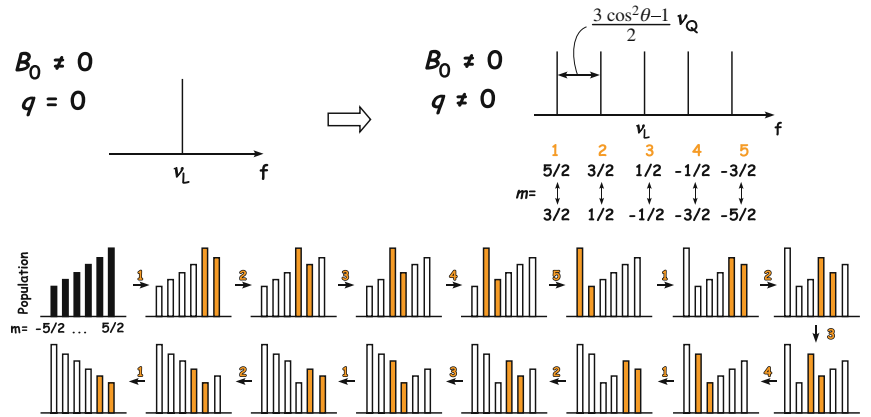

Fig. 1. Sequential AFP-NQR method. The upper part shows the schematic resonance frequencies under the electric quadrupole interaction, and they correspond to two-level transitions between $m$ and $m+1$. The lower part shows a sequence of AFP-r.f.s to invert the polarization.

where $\nu_{Q}$ was the quadrupole frequency $\nu_{Q} \equiv \frac{3}{2 I(2 I-1)} \frac{e q Q}{h}$ and $\theta$ was the angle between $B_{0}$ and the direction of $q$ which was parallel to the crystalline $c$-axis of $\mathrm{Al}_{2} \mathrm{O}_{3}$. In the present measurement, the $c$-axis was set perpendicular to $B_{0}\left(\theta=90^{\circ}\right)$. In eq. (3), the first-order perturbation of the quadrupole interaction was taken into account for simplicity, and the distances between the neighboring resonances were equivalent to $\left|\nu_{Q}\right| / 2\left(\theta=90^{\circ}\right)$. We actually applied these frequencies calculated within the second-order perturbation of the quadrupole interaction in order to correct the small deviation from the $\left|\nu_{Q}\right| / 2$ caused by the comparatively large $\nu_{Q}$ especially for $\theta=90^{\circ}$. To obtain a maximum asymmetry change, a sequential AFP-NQR method was employed. In the case of $q \neq 0$, the population of the neighboring two sub-lebels $m$ and $m+1$ was interchanged by an rf-field with the corresponding frequency $f_{m, m+1}$ with the AFP method. To flip the polarization completely, a set of five frequencies which were determined as a function of the $\nu_{Q}$ was applied sequentially, e.g., we applied fifteen rf-fields in the following order as $1 \rightarrow 2 \rightarrow 3 \rightarrow$ $4 \rightarrow 5 \rightarrow 1 \rightarrow 2 \rightarrow 3 \rightarrow 4 \rightarrow 1 \rightarrow 2 \rightarrow 3 \rightarrow 1 \rightarrow 2 \rightarrow 1$, as shown in the lower part of fig. 1 . The resonant asymmetry change was detected around the $\nu_{Q}=\frac{3}{2 I(2 I-1)} \frac{e q Q}{h}$ in a similar way to the $\beta$-NMR measurement.

\section{Results}

The obtained $\beta$-NMR spectrum of ${ }^{33} \mathrm{Al}$ in $\mathrm{Si}$ at room temperature (R.T.) at $B_{0}=0.5000 \mathrm{~T}$ was shown in fig. 2 . The strength of the rf-field was about 8 gauss and it satisfied the AFP condition. As is shown in fig. 2, the Larmor frequency obtained from the Gaussian fit was found to be consistent with that reported in ref. [12]. The degree of polarization of ${ }^{33} \mathrm{Al}$ multiplied by the asymmetry parameter $(A P)$ was determined as $|A P|=1.8(2) \%$ from the amplitude of the NMR spectrum of ${ }^{33} \mathrm{Al}$ in Si. The $\beta$-NQR spectrum of ${ }^{33} \mathrm{Al}$ in $\mathrm{Al}_{2} \mathrm{O}_{3}$ at R.T. was successfully obtained as shown in fig. 3. The maximum asymmetry change was observed between the scan width of $\nu_{Q}$ from $317 \mathrm{kHz}$ to $366 \mathrm{kHz}$ represented by dashed lines in fig. 3. The magnitude of the maximum asymmetry 


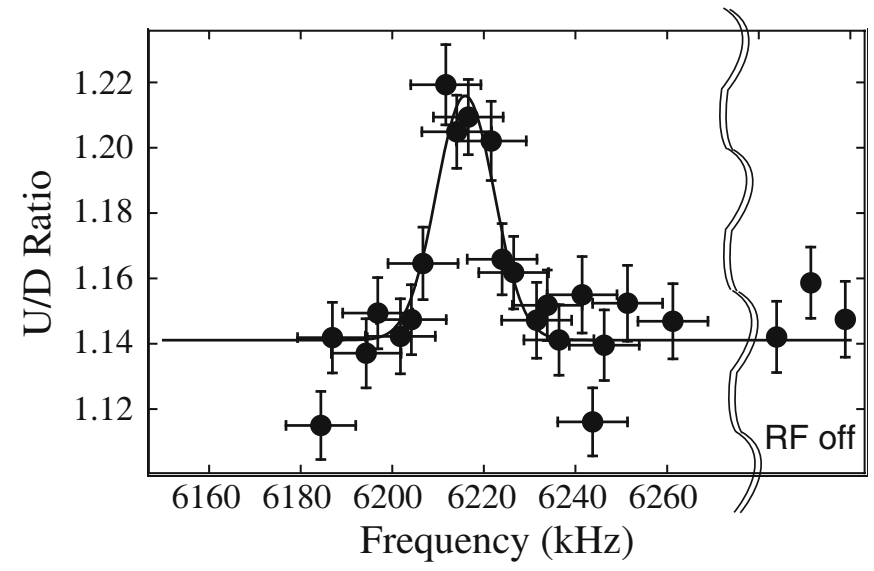

Fig. 2. Obtained NMR spectrum (points) of ${ }^{33} \mathrm{Al}$ in $\mathrm{Si}$ at room temperature together with the best-fit Gaussian curve.

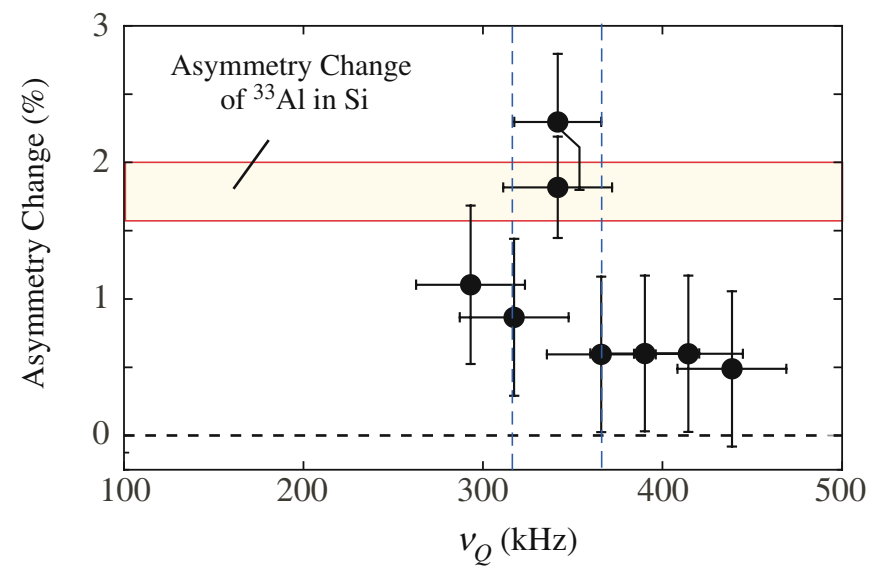

Fig. 3. Obtained NQR spectrum (points) of ${ }^{33} \mathrm{Al}$ in $\mathrm{Al}_{2} \mathrm{O}_{3}$ $\left(\theta=90^{\circ}\right)$ at room temperature together with the asymmetry change obtained by $\beta$-NMR on ${ }^{33} \mathrm{Al}$ in $\mathrm{Si}$ (band).

change was consistent with the polarization determined from the $\beta$-NMR of ${ }^{33} \mathrm{Al}$ in $\mathrm{Si}$. As a reference to extract $Q\left({ }^{33} \mathrm{Al}\right)$, we utilized the electric quadrupole coupling constant $e q Q$ of ${ }^{27} \mathrm{Al}$ in $\mathrm{Al}_{2} \mathrm{O}_{3}$ and $Q$ moment of ${ }^{27} \mathrm{Al}$, which were well determined as $e q Q / h\left({ }^{27} \mathrm{Al}\right)=2389(2) \mathrm{kHz}[15]$ and $\left|Q\left({ }^{27} \mathrm{Al}\right)\right|=146.6(10) \mathrm{emb}[16]$, respectively. The quadrupole moment of ${ }^{33} \mathrm{Al}$ is extracted from the following equation:

$$
\left|Q\left({ }^{33} \mathrm{Al}\right)\right|=\left|\frac{e q Q / h\left({ }^{33} \mathrm{Al}\right)}{e q Q / h\left({ }^{27} \mathrm{Al}\right)}\right| \times\left|Q\left({ }^{27} \mathrm{Al}\right)\right| .
$$

From fig. 3 and eq. (4), we obtained the preliminary $Q$ moment of ${ }^{33} \mathrm{Al}$ as $\left|Q\left({ }^{33} \mathrm{Al}\right)\right| \sim 130 \mathrm{emb}$. The theoretical predictions of $Q\left({ }^{33} \mathrm{Al}\right)$ are $118 \mathrm{emb}$ and $166 \mathrm{emb}$ with the conventional shell model and SDPF-M [14], respectively. From the present result, the contribution of the intruder configuration seems to be not so large as the SDPF-M prediction. To describe the details of the nuclear structure of ${ }^{33} \mathrm{Al}$ and to conclude whether the ${ }^{33} \mathrm{Al}$ isotope is located inside or outside of the island of inversion clearly, further analyses on the other NQR spectra obtained with narrower scan width compared to that as shown in fig. 3 are in progress.

\section{References}

1. C. Detraz et al., Phys. Rev. C 19, 164 (1979).

2. D. Guillemaud et al., Nucl. Phys. A 426, 37 (1984).

3. T. Motobayashi et al., Phys. Lett. B 346, 9 (1995).

4. E.K. Warburton, J.A. Becker, B.A. Brown, Phys. Rev. C 41, 1147 (1990).

5. G. Huber et al., Phys. Rev. C 18, 2342 (1978).

6. M. Keim et al., Eur. Phys. J. A 8, 31 (2000).

7. G. Neyens et al., Phys. Rev. Lett. 94, 022501 (2005).

8. D.T. Yordanov et al., Phys. Rev. Lett. 99, 212501 (2007).

9. H. Ueno et al., Phys. Lett. B 615, 186 (2005).

10. D. Nagae et al., Phys Rev. C 79, 027301 (2009).

11. D. Kameda et al., Phys. Lett. B 647, 93 (2007).

12. P. Himpe et al., Phys. Lett. B 643, 257 (2006).

13. P. Himpe et al., Phys. Lett. B 658, 203 (2008).

14. Y. Utsuno, private communication.

15. S.J. Gravina et al., J. Magn. Res. 89, 515 (1990).

16. V. Kellö et al., Chem. Phys. Lett. 304, 414 (1999). 